\title{
On singular lagrangian underlying the Schrodinger equation
}

\author{
Alexei Deriglazov* \\ UFJF, MG-Brazil \\ E-mail: alexei.deriglazov@ufjf.edu.br
}

\begin{abstract}
We analyze the properties that manifest hamiltonian nature of the Schrödinger equation and show that it can be considered as originating from singular Lagrangian action (with two second class constraints presented in the Hamiltonian formulation). It is used to show that any solution of the Schrödinger equation with time independent potential can be presented in the form $\Psi=\left(-\frac{\hbar^{2}}{2 m} \triangle+V\right) \phi+i \hbar \partial_{t} \phi$, where the real field $\phi\left(t, x^{i}\right)$ is some solution of nonsingular Lagrangian theory being specified below. Preservation of probability turns out to be the energy conservation law for the field $\phi$. After introduction the field into the formalism, its mathematical structure becomes analogous to those of electrodynamics.
\end{abstract}

5th International School on Field Theory and Gravitation April 20-24, 2009

Cuiabá city, Brazil

*Poster Section. On leave of absence from Dept. Math. Phys., Tomsk Polytechnical University, Tomsk, Russia. 
Introduction. Hamiltonian character of the Schrödinger equation is widely explored in various quantum mechanical applications [1]. In classical mechanics, Hamiltonian equations for the phase space variables $q, p$ normally originate from a Lagrangian formulation for the configuration variables $q$ : there exists an action that implies the second order equations equivalent to the Hamiltonian ones. It is the aim of this work to show that the Schrödinger equation with time independent potential admits a similar treatment.

In fact, the problem has been raised already by Schrödinger [2]. Eq. (5) below has been tested by Schrödinger as a candidate for the wave function equation and then abandoned. So, the real field $\phi$ appeared in this equation will be called the Schrödinger field. We establish the simple formula (4) that generates solutions of the Schrödoinger equation from solutions of the Schrödinger field equation. Then we present the singular Lagrangian theory that implies unified description for both the Schrödinger equation and the Schrödinger field equation. The unified formulation is used, in particular, to prove that any solution of the Schrödinger equation can be presented according to the formula (4). It implies, that after introduction the Schrödinger field into the formalism, its mathematical structure becomes analogous to those of electrodynamics. In particular, as well as $A^{\mu}$ represents a potential for magnetic and electric fields, the Schrödinger field turns out to be a potential for the wave function, giving its real and imaginary parts according to Eq. (4). Other similarities are summarized in the table at the end of this work.

Nonsingular Lagrangian associated with the Schrödinger equation. We restrict ourselves to the one-particle Schrödinger equation with time independent potential $V\left(x^{i}\right)$

$$
i \hbar \dot{\Psi}=-\triangle \Psi+V \Psi
$$

We use the notation $\triangle=\frac{\hbar^{2}}{2 m} \frac{\partial^{2}}{\partial x^{i 2}}, \vec{\nabla}=\frac{\hbar}{\sqrt{2 m}} \frac{\partial}{\partial x^{i}}, \dot{\varphi}=\frac{\partial \varphi\left(t, x^{i}\right)}{\partial t}$. It is equivalent to the system of two equations for two real functions (real and imaginary parts of the wave function $\Psi\left(t, x^{i}\right), \Psi=\varphi+i p$ )

$$
\hbar \dot{\varphi}=-(\triangle-V) p, \quad \hbar \dot{p}=(\triangle-V) \varphi
$$

Considering $p\left(t, x^{i}\right)$ as conjugate momenta for the field $\varphi\left(t, x^{i}\right)$, the system has the Hamiltonian form $\dot{\varphi}=\{\varphi, H\}, \dot{p}=\{p, H\}$, where $\{$,$\} stands for the Poisson bracket and H$ is the Hamiltonian $H=\frac{1}{2 \hbar} \int d^{3} x\left[\vec{\nabla} \varphi \vec{\nabla} \varphi+\vec{\nabla} p \vec{\nabla} p+V\left(\varphi^{2}+p^{2}\right)\right]$. Hence the equations (2) arise from the variation problem with Hamiltonian action obtained according to the known rule

$$
S_{H}=\int d t d^{3} x[p \dot{\varphi}-H]=\int d t d^{3} x\left[\frac{i \hbar}{2}\left(\Psi^{*} \dot{\Psi}-\dot{\Psi}^{*} \Psi\right)-\vec{\nabla} \Psi^{*} \vec{\nabla} \Psi-V \Psi^{*} \Psi\right]
$$

Following the classical mechanics prescription, to construct the Lagrangian formulation (if any) one needs to resolve the first equation from 2) with respect to $p$ and then to substitute the result either in the second one or into the Hamiltonian action (3). It leads immediately to rather formal nonlocal expression $p=-\hbar(\triangle-V)^{-1} \dot{\varphi}$. So, the Schrödinger system can not be obtained starting from some (nonsingular) Lagrangian. Nevertheless, there exists nonsingular Lagrangian field theory with the property that any solution of the Schrödinger equation can be constructed from some solution of this theory. To find it let us look for solutions of the form

$$
\Psi=-(\triangle-V) \phi+i \hbar \dot{\phi},
$$


where $\phi\left(t, x^{i}\right)$ is some real function. $\Psi$ will be solution of the Schrödinger equation if $\phi$ obeys the equation

$$
\hbar^{2} \ddot{\phi}+(\triangle-V)^{2} \phi=0,
$$

the latter follows from the Lagrangian action

$$
S[\phi]=\int d t d^{3} x\left[\frac{\hbar}{2} \dot{\phi} \dot{\phi}-\frac{1}{2 \hbar}[(\triangle-V) \phi]^{2}\right] .
$$

It is considered here as the classical theory of field $\phi$ on the given external background $V\left(x^{i}\right)$. The action involves the Planck's constant as a parameter. After the rescaling $\left(t, x^{i}, \phi\right) \rightarrow\left(\hbar t, \hbar x^{i}, \sqrt{\hbar} \phi\right)$ it appears in the potential only, $V\left(\hbar x^{i}\right)$, and thus play the role of coupling constant of the field $\phi$ with the background.

According to the formula (4), both probability density and phase of the wave function can be presented through the Schrödinger field. Taking $\Psi=\sqrt{P} \exp \frac{i}{\hbar} S$ one obtains $P=\hbar^{2}(\dot{\phi})^{2}+[(-\triangle+$ $V) \phi]^{2}=2 \hbar E, S=-\hbar \arctan \sqrt{\frac{T}{U}}$, where $E=T+U$ is energy density of the Schrödinger field. The first equation states that the probability density is the energy density of the Schrödinger field. Invariance of the action under the time translations implies the current equation $\partial_{t} E+\vec{\nabla}\left(2 \hbar^{-2} E \vec{\nabla} S\right)=0$. Thus preservation of probability is just the energy conservation law of the theory (6).

It is instructive to compare also the Hamiltonian equations of the theory (6)

$$
\hbar \dot{\phi}=p, \quad \hbar \dot{p}=-(\triangle-V)^{2} \phi,
$$

with the Schrödinger system. One notes the following correspondence among solutions of these systems: a) If the functions $\varphi, p$ obey Eq. (2), then the functions $\phi \equiv \varphi,-(\triangle-V) p$ obey (7). b) If the functions $\phi, p$ obey Eq. (7), then $\varphi \equiv-(\triangle-V) \phi, p$ obey (2). Kernel of the map $(\varphi, p) \rightarrow(\phi, p)$ is composed by pure imaginary time independent wave functions $\Psi=i \Pi\left(x^{i}\right)$, where $\Pi$ is any solution of the stationary Schrödinger equation $(\triangle-V) \Pi=0$.

Any solution of the field theory (6) determines some solution of the Schrödinger equation according to Eq. (4). Then one should ask whether an arbitrary solution of the Schrödinger equation can be presented in the form (4)? An affirmative answer will be obtained in the next section using the Dirac approach to description the constrained systems [3]. Besides, in this setting one obtains more systematic treatment of the observations made above: there exists the singular Lagrangian theory subject to second class constraints underlying both the Schrödinger equation and the classical theory (6).

Our appeal to the constrained theories can be motivated as follows. Treatment of the Schrödinger system (2) as the Hamiltonian one does not allow one to construct the corresponding Lagrangian formulation owing to presence the spatial derivatives of momenta in the Hamiltonian. To avoid the problem, let us try to treat the Schrödinger system as a generalized Hamiltonian system. Namely, one rewrites (2) in the form $\dot{\varphi}=\left\{\varphi, H^{\prime}\right\}^{\prime}, \dot{p}=\left\{p, H^{\prime}\right\}^{\prime}$, where $H^{\prime}$ is the "free field" generalized Hamiltonian $H^{\prime}=\int d^{3} x \frac{1}{2 \hbar}\left(p^{2}+\varphi^{2}\right)=\int d^{3} x \frac{1}{2 \hbar} \Psi^{*} \Psi$, and the non canonical Poisson bracket is ${ }^{1}$

$$
\{\varphi, \varphi\}^{\prime}=\{p, p\}^{\prime}=0, \quad\{\varphi(t, x), p(t, y)\}^{\prime}=-(\triangle-V) \delta^{3}(x-y) .
$$

\footnotetext{
${ }^{1}$ In classical mechanics, inclusion of an interaction into a symplectic structure of the phase manifold has been investigated by Souriau [4]. It is discussed also in the framework of non commutative theories [5]. Similar construction is known for the Maxwell equations. They have been recognized as the generalized Hamiltonian equations in [6].
} 
In contrast to $H$, the Hamiltonian $H^{\prime}$ does not involve the spatial derivatives of momentum.

Non canonical bracket turns out to be a characteristic property of the theories with second class constraints. In this case the constraints can be taken into account by transition from the Poisson to the Dirac bracket, the latter represents an example of non canonical bracket. Hamiltonian equations for dynamical variables, being written in terms of the Dirac bracket, form a generalized Hamiltonian system. So, the equations (8) represent a hint to search for associated constrained Lagrangian.

Singular Lagrangian underlying the Schrödinger equation. Here we obtain (2) as the Hamiltonian equations that follow from the Lagrangian action

$$
S[\phi, \varphi]=\int d t d^{3} x\left[\frac{\hbar}{2} \dot{\phi} \dot{\phi}+\frac{1}{2 \hbar} \varphi^{2}+\frac{1}{\hbar} \varphi(\triangle-V) \phi\right],
$$

written for two real fields $\phi\left(t, x^{i}\right), \varphi\left(t, x^{i}\right)$ on the given external background $V\left(x^{i}\right)$. It implies the Lagrangian equations $\hbar^{2} \ddot{\phi}-(\triangle-V) \varphi=0, \quad \varphi=-(\triangle-V) \phi$. As a consequence, both $\phi$ and $\varphi$ obey the second order equation (5). After the shift $\tilde{\varphi} \equiv \varphi+(\triangle-V) \phi$, the action acquires the form $S[\phi, \varphi]=S[\phi]+\frac{1}{2 \hbar} \int \tilde{\varphi}^{2}$. Hence in this parametrization the fields $\phi$ and $\tilde{\varphi}$ decouple, and the only dynamical variable is $\phi$. Its evolution is governed by Eq. (5). Being rather natural, it is not unique possible parametrization of dynamical sector. To find another relevant parametrization, we would like to construct Hamiltonian formulation of the theory. One introduces the conjugate momenta $p, \pi$ for the fields $\phi, \varphi$ and defines their evolution according to $p=\frac{\partial L}{\partial \phi}=\hbar \dot{\phi}, \quad \pi=\frac{\partial L}{\partial \dot{\varphi}}=0$. The second equation does not contain time derivative of the fields, hence it represents primary constraint of the theory. Then the Hamiltonian is

$$
H=\int d^{3} x\left[\frac{1}{2 \hbar}\left(p^{2}-\varphi^{2}\right)-\frac{1}{\hbar} \varphi(\triangle-V) \phi+v \pi\right]
$$

where $v$ stands for the Lagrangian multiplier of the constraint. Preservation in time of the primary constraint, $\dot{\pi}=\{\pi, H\}=0$, implies the secondary one $\varphi+(\triangle-V) \phi=0$. In turn, its preservation in time determines the Lagrangian multiplier $v=-\frac{1}{\hbar}(\triangle-V) p$. Hence the Dirac procedure stops on this stage. Evolution of the phase space variables is governed by the Hamiltonian equations

$$
\dot{\phi}=\frac{1}{\hbar} p, \quad \dot{p}=\frac{1}{\hbar}(\triangle-V) \varphi, \quad \dot{\varphi}=v \approx-\frac{1}{\hbar}(\triangle-V) p, \quad \dot{\pi} \approx 0,
$$

and by the constraints

$$
\pi=0, \quad \varphi+(\triangle-V) \phi=0 .
$$

The system implies that both $\phi$ and $\varphi$ obey Eq. (5). Computing the Poisson bracket of the constraints, one obtains on-shell non vanishing result $\{\varphi+(\triangle-V) \phi, \pi\}=\delta^{3}(x-y)$. According to the Dirac terminology, the constraints form a second class system.

We reminded the Dirac prescription for dealing with the second class constraints. They are used to determine a part of variables in terms of others. The variables that have been thus determined are conventionally called the non dynamical variables. Evolution of the remaining dynamical variables is governed by equations of first order with respect to time. They are obtained from the initial equations (11) taking into account the constraints as well as equations for the Lagrangian 
multipliers. There is an equivalent way to obtain the equations of motion for dynamical variables. One can write the Hamiltonian (10) in terms of dynamical variables, and to construct the Dirac bracket corresponding to the constraints (12)

$$
\{A, B\}_{D}=\{A, B\}-\{A, \pi\}\{\varphi+(\triangle-V) \phi, B\}+\{A, \varphi+(\triangle-V) \phi\}\{\pi, B\} .
$$

It implies $\{\pi, A\}=0,\{\phi, \varphi\}=0$, as well as

$$
\begin{gathered}
\{\phi, p\}_{D}=\delta^{3}(x-y), \quad\{\phi, \phi\}_{D}=\{p, p\}_{D}=0 ; \\
\{\varphi, p\}_{D}=-(\triangle-V) \delta^{3}(x-y), \quad\{\varphi, \varphi\}_{D}=\{p, p\}_{D}=0 .
\end{gathered}
$$

Then equation of motion for any dynamical variable $z_{d y n}$ can be written as follows:

$$
\dot{z}_{d y n}=\left\{z_{d y n}, H\left(z_{d y n}\right)\right\}_{D}
$$

It should be noticed that for the pair $\phi, p$ the Dirac brackets coincide with the Poisson ones. For the pair $\varphi, p$ the Dirac brackets coincide exactly with the non canonical ones (8).

Let us apply the prescription to the model under consideration. The constraints (12) imply that either $\phi, p$ or $\varphi, p$ can be chosen to parameterize the dynamical sector of the theory.

Parameterizing it by the pair $\varphi, p$, the equations (11) reduce to the Schrödinger system (2), while the Hamiltonian (10) acquires the form of "free field" Hamiltonian. Note that $p$ is the conjugate momenta for $\phi$ but not for $\varphi$. Using this Hamiltonian and the Dirac bracket (15), the equations (2) can be obtained also according to the rule (16).

Parameterizing the dynamical sector by the pair $\phi, p$, the equations (11) reduce to the system (7), while the Hamiltonian (10) acquires the form

$$
H(\phi, p)=\int d^{3} x \frac{1}{2 \hbar}\left[p^{2}+[(\triangle-V) \phi]^{2}\right] .
$$

It is just the Hamiltonian of the theory (6).

Hence the classical field theory (6) and the Schrödinger equation can be identified with two possible parameterizations of dynamical sector of the singular Lagrangian theory (9). Specifying parametrization one arrives at either classical or quantum description.

Let us return to the proof of the formula (4). Let $\Psi=\varphi+i p$ be a solution of the Schrödinger equation (1). Then there exists the function $\phi\left(t, x^{i}\right)$ such that $\varphi, p, \phi, \pi=0$ is a solution of the system (11), (12). Actually, except the first and the last equation, all other equations of the system are already satisfied. The remaining equations with known right hand sides $\dot{\phi}=\frac{1}{\hbar} p, \quad(\triangle-V) \phi=$ $-\varphi$, specify the function $\phi$. Take the second equation at $t=0,(\triangle-V) \phi=-\varphi\left(0, x^{i}\right)$. The elliptic equation can be solved (at least for the analytic function $\varphi\left(x^{i}\right)$ ), let us denote the solution as $C\left(x^{i}\right)$. Then the function $\phi\left(t, x^{i}\right)=\frac{1}{\hbar} \int_{0}^{t} d \tau p\left(\tau, x^{i}\right)+C\left(x^{i}\right)$, obeys both these equations. They imply the desired result: the wave function can be presented through the field $\phi$ and its momenta according to (4).

Conclusion In this work we have associated the classical field theory (6) with quantum mechanics of a particle in time independent potential. It has been shown that the Schrödinger equation is mathematically equivalent to the Schrödinger field equation (5). Solving the classical theory, one 


\begin{tabular}{|l|l|}
\hline Electrodynamics & Quantum mechanics \\
\hline $\begin{array}{l}\text { There is the Lagrangian formulation in terms } \\
\text { of } A^{\mu}\end{array}$ & The same in terms of $\phi$ \\
\hline $\begin{array}{l}A^{\mu} \text { represents the potential for magnetic and } \\
\text { electric fields, in the gauge } A^{0}=0 \text { one has } \\
\vec{B}=\nabla \times \vec{A}, \vec{E}=\partial_{t} \vec{A}\end{array}$ & $\Psi=\varphi+i p=-(\triangle-V) \phi+i \hbar \partial_{t} \phi$ \\
\hline $\begin{array}{l}\text { While the Maxwell equations are written in } \\
\text { terms of } \vec{B}, \vec{E} \text {, the field } \vec{E} \text { is the conjugate mo- } \\
\text { menta for } \vec{A} \text { but not for } \vec{B}\end{array}$ & $\begin{array}{l}\text { While the Schrödinger equation is written in } \\
\text { terms of } \varphi, p \text {, the field } p \text { is the conjugate mo- } \\
\text { menta for } \phi \text { but not for } \varphi\end{array}$ \\
\hline $\begin{array}{l}\text { Maxwell equations form the generalized } \\
\text { Hamiltonian system [6] with the Hamiltonian }\end{array}$ & $\begin{array}{l}\text { Schrödinger equation forms the generalized } \\
\text { Hamiltonian system with the Hamiltonian } ~ \\
\sim \vec{E}^{2}+\vec{B}^{2}\end{array}$ \\
\hline
\end{tabular}

Figure 1: Schrödinger field $\phi$ as the wave function potential.

is able to construct the quantum mechanical object, $\Psi$, according to the formula (4). The later may be considered as a kind of quantization rule $\phi_{\text {class }} \longrightarrow \Psi_{Q M}=D \phi+i p$, where $p$ is conjugate momenta for $\phi$ and $D$ stands for some differential operator specified by the theory. Origin of the rule has been explained in the Section 3: either the classical field or quantum mechanical equations result from two possible parameterizations of dynamical sector of the singular theory (9).

While we have started with quantum mechanics and arrived at a field theory, the procedure can be inverted. It would be interesting to apply it to other (relativistic) field theories.

To conclude with, it should be noticed that the presented formulation implies certain analogy among mathematical structure of the Schrödinger equation and the free electrodynamics, see the Fig. 1 above. Roughly speaking, the Schrödinger field turns out to be the wave function potential. Acknowledgments. Author would like to thank A. Nersessian for encouraging discussions. The work has been supported by the Brazilian foundations CNPq and FAPERJ.

\section{References}

[1] G. Wentzel, Z. Phys. 38 (1926) 518; H. A. Kramers, Z. Phys. 39 (1926) 828; L. Brillouin , J. Phys. 7 (1926) 353; V. P. Maslov and M. V. Fedoriuk, Semi-classical approximation in quantum mechanics (Dordrecht: D. Reidel Publishing Co., 1981); D. Bohm, Phys. Rev. 85 (1952) 166; ibid 180.

[2] E. Schrödinger, Ann. Physik 81 (1926) 109; see also letters by Shrödinger to Lorentz in: K. Przibram, Briefe zür Wellenmechanik, (Wien, 1963).

[3] P.A.M. Dirac, Can. J. Math. 2 (1950) 129; Lectures on Quantum Mechanics (Yeshiva Univ., New York, 1964); D. M. Gitman and I. V. Tyutin, Quantization of Fields with Constraints (Berlin: Springer-Verlag, 1990); A. A. Deriglazov, Phys. Lett. B 626 (2005) 243-248.

[4] J. M. Souriau, Structure des Systemes Dynamiques (Dund, Paris, 1970).

[5] A. A. Deriglazov, Phys. Lett. B530 (2002) 235; JHEP 0303 (2003) 021.

[6] W. Pauli (1933), General Principles of Quantum Mechanics, (reprinted in English translation by Springer, 1981); M. Born and L. Infeld. Proc. Roy. Soc. A 150 (1935) 141. 